Voix et Images

voixetimages

\title{
Rencontres et solitude
}

\section{Martine-Emmanuelle Lapointe}

Volume 37, numéro 1 (109), automne 2011

URI : https://id.erudit.org/iderudit/1006468ar

DOI : https://doi.org/10.7202/1006468ar

Aller au sommaire du numéro

\section{Éditeur(s)}

Université du Québec à Montréal

\section{ISSN}

0318-9201 (imprimé)

1705-933X (numérique)

Découvrir la revue

Citer ce compte rendu

Lapointe, M.-E. (2011). Compte rendu de [Rencontres et solitude]. Voix et Images, 37(1), 137-141. https://doi.org/10.7202/1006468ar d'utilisation que vous pouvez consulter en ligne.

https://apropos.erudit.org/fr/usagers/politique-dutilisation/ 


\section{RO M A N}

Rencontres et solitude

$+\quad+\quad+$

MARTINE-EMMANUELLE LAPOINTE

Université de Montréal

Dans Voyage au Maghreb en l'an mil quatre cent de l'Hégire ${ }^{1}$, Louis Gauthier renoue le fil de ses précédents récits de voyage, publiés sous les titres Voyage en Irlande avec un parapluie, Le pont de Londres et Voyage au Portugal avec un Allemand ${ }^{2}$. Comme dans les premiers volumes de la tétralogie, Voyage au Maghreb met en scène un voyageur qui relate les expériences, les rencontres et les menus bonheurs et désagréments qui traversent sa vie d'errance. L'histoire peut sembler banale: quelques mois avant le référendum de 1980, un homme quitte le Québec pour soigner un chagrin d'amour. Au fil de ses déplacements, il adresse des lettres - imaginées dans le vif de ses aventures - à Angèle, la femme qui l'a quitté. Si l'intrigue est minimale, attachée presque entièrement au discours d'une conscience, elle n'en est pas pour autant narcissique. Le narrateur se regarde avec une certaine distance, refuse la complaisance, ose mettre en doute ses moindres certitudes en les confrontant aux opinions des autres. Sa quête et sa destination ultimes - l'Inde - ne sont que prétextes à une lente redécouverte de soi. Celui qui cherchait à être ébloui, attendant un miracle, en vient à mieux comprendre ce qui l'anime et renonce par là même à conférer un sens à son voyage. Dans une lettre à Angèle, il écrit: "ce matin je me suis réveillé avec, pendant un bref moment, la sensation que je n'étais qu'un événement du monde, et cela m’a apaisé. Je n'avais pas à tout comprendre ni à tout contrôler, je n'étais qu'une petite partie sans importance qui participait au tout» (112). Ce passage n'est pas sans faire écho aux paroles de la mystique sainte Thérèse, « [s] oyez ce que vous êtes et le monde s'enflammera» (158), citées par un jeune hippie autrichien croisé à Kairouan.

Loin de célébrer le pittoresque du Maghreb, le récit de voyage est plutôt l'occasion d'une réflexion sur l'amitié, sur le choc provoqué par toute rencontre, fût-elle uniquement avec soi. S'il croise de nombreux compagnons de route, le narrateur n'en demeure pas moins solitaire, le plus souvent incapable d'être «dans le même voyage que les autres» (54), se demandant même: «et si on n'a pas envie de rencontrer les

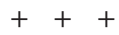

1 Louis Gauthier, Voyage au Maghreb en l'an mil quatre cent de l'Hégire, Montréal, Fides, 2011, 187 p. 2 La trilogie a été publiée en un seul volume en 2005 sous le titre Voyage en Inde avec un grand détour (Montréal, Fides, 274 p.). 
autres? » (47) Le fait de ne pas rencontrer les autres ne constitue pas tant un projet qu'une exigence intérieure. Taciturne, le narrateur se juge inintéressant, a du mal à jouer un rôle, à se prendre pour un écrivain et un voyageur, d'où sans doute l'impression de modestie que laisse au lecteur le récit de Gauthier. Même s'il ne cesse de faire retour sur son passé et sur ses insuffisances, le narrateur ne cède jamais à la prétention.

Dès la parution du Voyage en Irlande avec un parapluie en 1984, la critique a évoqué le caractère sobre, sans ostentation, du style de Louis Gauthier. En lisant les récits de l'auteur, on pourrait croire que rien ne s'y passe. Il s'agit bien sûr d'une fausse impression que démentent plusieurs scènes et descriptions. Le récit progresse lentement - ce qui n'est nullement un tort - et s'attache par moments à des images fulgurantes, scorpions forniquant dans le désert, doubles de Marie et de Joseph croisés dans le train, vision apocalyptique d'une plateforme pétrolière. La prose dépourvue d'artifices de Louis Gauthier exige ainsi la participation de ses lecteurs. Un peu à l'image du narrateur, elle n'est pas plaisante ni séduisante, mais austère et dépouillée, qualités plutôt rares dans la littérature contemporaine. Le dépaysement ressenti par le lecteur ne naît donc pas de l'exotisme des lieux et des situations, mais de la facture presque inactuelle du Voyage au Maghreb en l'an mil quatre cent de l'Hégire.

La littérature joue également un rôle important dans l'œuvre de Louis Gauthier. Écrivain en devenir, le narrateur est d'abord et avant tout un lecteur, un herméneute parfois davantage intéressé par les mondes fictifs que par la réalité. C'est en lisant Sur la route de Jack Kerouac qu'il se sent américain : «je m’aperçois que j'aime l'Amérique ; tout à coup j'éprouve une grande envie d'y être, une grande envie de cette liberté, de cette créativité, de ce dynamisme» (129), comme si la littérature le ramenait à ses origines plutôt que de l'en éloigner.

Le dernier roman d'Abla Farhoud, Le sourire de la petite juive ${ }^{3}$, se présente sous la forme d'une cartographie romanesque de la rue Hutchison. Sorte de mise en abyme, le roman met en scène l'écrivaine Françoise Camirand, qui habite dans cette rue depuis trenteneuf ans. À l'instar d'Abla Farhoud, elle choisit d'écrire un livre sur la vie de son quartier composé des portraits de ses voisins. Le titre de l'œuvre témoigne d'une obsession assez courante dans les romans qui se déroulent dans le Mile End ou à Outremont, soit le désir d'une rencontre avec les membres de la communauté juive hassidique. Le mystère de ce groupe autosuffisant, vivant en marge du monde des autres Montréalais, fascine et irrite de nombreux personnages de fiction. Il y aurait sans doute lieu d'interroger ou de mettre en perspective cet intérêt pour la communauté hassidique. Pourquoi intriguet-elle autant? Peut-être parce qu'elle représente l'une des formes d'altérité les plus radicales qui soit... À l'heure de la laïcité, cette communauté se définit littéralement en fonction de sa pratique et de ses rituels. Coiffures et costumes singuliers, mezouza, lieux de culte distincts sont autant de signes ostentatoires d'une croyance qui éloignent

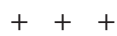

3 Abla Farhoud, Le sourire de la petite juive, Montréal, VLB éditeur, coll. «Fictions», 2011, 209 p.

VOIX ET IMAGES $109 \quad \mathbf{1 3 8}$ 
l'étranger et confirment la solidarité des membres du groupe. Tant dans Babel prise deux ou Nous avons tous découvert l'Amérique de Francine Noël que dans Hadassa de Myriam Beaudoin, eux aussi campés non loin de la rue Hutchison, les personnages principaux souhaitent entrer en contact avec les hassidim, obtenir ne serait-ce qu'un sourire afin d'être reconnus par leurs voisins. C'est d'ailleurs sur une note d'espoir que se termine le roman de Francine Noël, publié en 1990:

\begin{abstract}
Il faisait doux cet après-midi, j'étais au coin de la rue, à contempler un magnifique 101 sur fond rouge, quand j'ai aperçu la grand-mère hassidique d'à côté. Elle rentrait chez elle avec son cabas. Elle était seule. Je lui ai souri comme d'habitude, et c'est alors que quelque chose s'est produit: ELLE M'A SOURI. En me regardant droit dans les yeux. Mais vraiment souri! Puis, elle a passé son chemin. Il n'y avait personne autour de nous ${ }^{4}$.
\end{abstract}

À cet égard, l'originalité du roman d'Abla Farhoud réside dans la mise en scène de la jeune Hinda Rochell qui est fascinée par la langue française et par l'œuvre de Gabrielle Roy. Des pages de son journal intime, rédigées en français, sont intégrées au roman et dévoilent ses pensées secrètes, celles qu'elle ne saurait communiquer aux membres de sa famille immédiate. Scellant symboliquement la réconciliation des communautés, la rencontre entre la petite juive du titre et l'écrivaine Françoise Camirand a lieu vers la fin du roman. Dans son journal, Hinda relate ce moment singulier:

Je l'ai vue souvent cette femme-là. L'été, elle s'habille toujours en blanc. Et l'hiver en noir. Je l'ai déjà vue sourire aux petits enfants de notre communauté, mais pas aux filles de mon âge ni aux adultes. Avant de me parler, elle m'a souri. J'étais à deux pas de la maison. Je l'ai vue traverser la rue et venir vers moi. Son sourire m’a trop surprise. Je n'ai pas souri (169).

Comme en témoigne ce passage, le roman laisse peu de place au non-dit; au contraire, il explique, justifie, affiche une transparence trop parfaite. La cohérence de l'ensemble repose sur la présence de l'écrivaine au travail, qui s'interroge sur ses voisins, les épie et les invente. Le procédé devient lassant. Tout se passe comme si Abla Farhoud souhaitait colmater les brèches de la vie communautaire montréalaise en ne privilégiant que le bon côté des choses. Son roman est vertueux, presque trop empathique, et n'arrive pas à traduire la complexité des relations entre groupes culturels.

Le sablier des solitudes ${ }^{5}$ de Jean-Simon Desrochers emprunte lui aussi la forme d'un roman choral, mais, contrairement au Sourire de la petite juive, il ne donne guère dans les bons sentiments. L'auteur s'intéresse plutôt aux perversions et aux obsessions des

$$
+++
$$

4 Francine Noël, Babel prise deux ou Nous avons tous découvert l'Amérique, Montréal, VLB éditeur, 1990, p. 369.

5 Jean-Simon Desrochers, Le sablier des solitudes, Montréal, Les Herbes rouges, 2011, 359 p. 
treize personnages mis en scène, dont un militaire, un ministre, un avocat, une agente immobilière et un chauffeur de camion ou d'autocar, obsédés par la pornographie, l'ésotérisme ou le culte du corps. Le roman, composé de courts chapitres consacrés aux différents protagonistes, vise à dévoiler la psyché des êtres en observant leurs manies et leurs tics. Les treize personnages se croisent dans des circonstances tragiques, lors d'un terrible carambolage survenant au cœur de l'hiver. La catastrophe sert ainsi de ressort dramatique et confère une cohérence à un récit qui pouvait sembler décousu de prime abord. C'est que le principe d'alternance des différents personnages ne donne pas lieu à une véritable polyphonie. Chacun reste enfermé dans son petit univers capsule, coupé des autres, portant une blessure ou un handicap particuliers. Certes, cette stratégie permet de révéler l'irréparable solitude des protagonistes, mais elle a également le tort de figer leurs personnalités, comme si chacun répondait à un type. Le microcosme social recréé par Jean-Simon Desrochers a parfois des allures d'inventaire des vices et des vertus du siècle. Nous ne sommes pas chez Balzac bien sûr, enfin pas tout à fait. La multiplication des personnages - déjà présente dans le premier roman de l'auteur, La canicule des pauvres ${ }^{6}$, paru en $2009-$ dévoile cependant un désir d'exhaustivité, voire d'objectivité que l'on retrouve peu dans le roman contemporain.

Comme dans le roman de Louis Gauthier, la littérature joue ici un rôle structurant. Certains des exergues sont empruntés aux œuvres d'écrivains états-uniens, Raymond Carver et Russell Banks notamment, et inscrivent l'écriture de Desrochers dans une filiation nord-américaine. Comme chez Carver et Banks, les lieux romanesques du Sablier des solitudes sont dépourvus de poésie, souvent ordinaires et sans âme. Les banlieues dortoirs, les centres commerciaux, les stationnements, les routes secondaires se succèdent. Grâce à ses descriptions cliniques et à la précision de son style, Desrochers parvient à en cerner le caractère inhospitalier sans pour autant insister trop lourdement sur leur vacuité et leur laideur. En témoigne la description du centre commercial où se croiseront furtivement certains des personnages du roman :

En ce 4 janvier de froid polaire, il y a foule aux Promenades. Des familles entières déambulent sans rythme. De jeunes mères poussent le carrosse de leurs nourrissons joufflus, des enfants piquent des crises devant des magasins de jouets. De jeunes hommes branchés évaluent les rabais sur les vêtements griffés. Des plus vieux marchent à côté de leur matrone de banlieue, le regard neurasthénique, vide comme un cercueil à vendre. Des hardes de jeunes filles pimpantes boivent des Red Bull en caquetant. D'autres personnes, solitaires comme Lydia, arpentent le mail sans autre but que d'accélérer ce temps des fêtes qui ne fête plus que son immédiate nostalgie (161).

Ici encore l'auteur présente une vue d'ensemble, passe d'une conscience à l'autre, d'un corps à l'autre. Dans Le sablier des solitudes, le désœuvrement se porte comme

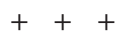

6 Jean-Simon Desrochers, La canicule des pauvres, Montréal, Les Herbes rouges, 2009, 672 p. 
un vêtement, accompagne, mine de rien, les contemporains dans leurs moindres déplacements. Comme dans la poésie unanimiste de Jules Romains, la foule a une âme, elle est traversée d'une même "immédiate nostalgie». Il faut un talent certain pour ainsi traduire les tropismes qui animent la communauté sans donner dans la caricature. Ce talent, Jean-Simon Desrochers le possède sans nul doute. 